Tensiones económicas, politicas y sociales durante los gobiernos de Evo Morales. Una interpretación sobre el golpe de Estado Alejandro M. Schneider

\title{
Tensiones económicas, politicas y sociales durante los gobiernos de Evo Morales. Una interpretación sobre el golpe de Estado
}

\author{
Economic, Political and Social Tensions during the \\ Governments of Evo Morales. An Interpretation of the \\ Coup d' Etat
}

\author{
ALEJANDRO M. SCHNEIDER \\ Facultad de Humanidades y Ciencias de la Educación; \\ Universidad Nacional de La Plata; \\ Centro de Investigaciones Socio-históricas (Argentina) \\ aschneider98@yahoo.com.ar
}

RESUMEN

El presente artículo busca analizar y reflexionar sobre una serie de escenarios conflictivos entre los distintos movimientos sociales y los gobiernos de Evo Morales Ayma en Bolivia, los cuales anticipan y explican en gran medida el golpe de Estado de noviembre de 2019. En ese sentido, en un primer momento, el ensayo busca exponer las principales características que tuvo la política económica del Movimiento Al Socialismo (MAS) en los trece años de mandato presidencial. En una segunda parte, se aborda la cambiante relación mantenida entre los diferentes movimientos sociales y la administración de Morales; en particular, se observan un conjunto de protestas que se dieron alrededor de sus medidas económicas. Ambas cuestiones permitirán comprender el origen de las tensiones politicas experimentadas en las semanas previas a su derrocamiento.

Palabras clave: Evo Morales; Bolivia; Movimientos Sociales; Golpe de Estado

ABSTRACT

This article seeks to analyze and reflect on a series of conflicting scenarios between the different social movements and the governments of Evo Morales Ayma in Bolivia, which largely anticipate and explain the coup d' Etat of November 2019. In this sense, at first, the essay seeks to expose the main characteristics of the economic policy of the Movement Towards Socialism (MAS) in the thirteen years of the presidential mandate. In a second part, deals with the changing relationship between the different 
Tensiones económicas, políticas y sociales durante los gobiernos de Evo Morales. Una interpretación sobre el golpe de Estado

social movements and the Morales administration; in particular, there is a set of protests that took place around their economic measures. Both issues will help to understand the origin of the political tensions experienced in the weeks prior to his overthrow.

Keywords: Evo Morales; Bolivia; Social Movements; Coup d' Etat

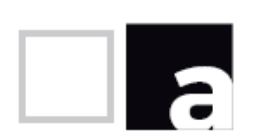

\section{Introducción}

En el convulsionado año de 1985 emergió una nueva Bolivia. El país que nació con la Revolución de 1952 comenzó un proceso de modificaciones estructurales. El ascenso al poder del líder histórico del Movimiento Nacionalista Revolucionario (MNR) Víctor Paz Estensoro marcó el cierre de una etapa, en la que él fue un protagonista destacado. A partir de entonces, con el Decreto Supremo (DS) 21060 se abrió un ciclo político, económico y social signado por una fuerte reestructuración capitalista sobre la sociedad.

Tras proclamar que "Bolivia se nos muere", Paz inauguró un drástico programa de medidas económicas, que acarreó un nuevo acuerdo de dominación sobre la sociedad civil. Se buscó recrear y restablecer la autoridad estatal perdida en los últimos años, en particular, sobre la clase obrera y el campesinado. En ese escenario, el mundo de la minería expresó los cambios más radicales: en el plazo de doce meses, se cerraron más de cincuenta minas estatales, aumentando las tasas de desocupación y subempleo, convirtiendo en pueblos fantasmas a numerosos distritos erigidos alrededor del trabajo del subsuelo. A la par de este fenómeno, como parte del modelo económico implementado, fue creciendo el sector informal a través del comercio al menudeo y de los servicios. Además, el proceso productivo se orientó hacia diversos sectores, entre ellos, el cultivo de la coca, la minería privada, etc. En otras palabras, desde entonces se fue configurando un mundo laboral distinto al producido por la Revolución de 1952.

Aunque la presencia militante del proletariado minero disminuyó, el ciclo de rebeliones abierto a partir de las guerras del agua (2000) y del gas (2003) junto con

\section{anuario.}


la llegada al gobierno del Movimiento al Socialismo (MAS) volvió a instalar a los mineros en la escena política. Ahora bien, no se encontraban solos. Al calor de distintas demandas y protestas, en los años noventa, otros protagonistas se convirtieron en actores centrales del presente milenio: campesinos, cocaleros, indigenas, cooperativistas, etcétera.

El ascenso a la presidencia de Evo Morales Ayma, en enero de 2006, en gran medida fue producto tanto de numerosas oleadas de luchas y reclamos como de la emergencia de esos colectivos sociales que se expresaron en distintas organizaciones. Aunque sus administraciones lograron una cierta redistribución de la riqueza, el escenario económico y social no se modificó en sus aspectos sustanciales. Las consecuencias de la reestructuración capitalista implementada en las décadas de los ochenta y noventa del siglo pasado todavía permanecen en el horizonte boliviano. A pesar de ello, en los comicios de 2009 y de 2014, una gran parte de la ciudadanía renovó su confianza en el mandatario campesino. Sin embargo, lentamente comenzó un deterioro en su ejercicio de gobierno; la derrota electoral en febrero de 2016 fue una clara advertencia que los tiempos estaban cambiando.

En ese escenario de permanente transformación, el presente artículo busca analizar y reflexionar sobre una serie de horizontes conflictivos que se plantearon en esas presidencias; los cuales anticipan y explican en gran medida el golpe de Estado de noviembre de 2019. En la primera parte de este estudio se indagará sobre los principales lineamientos económicos durante esos trece años de gobierno; posteriormente, el examen se detendrá en la versátil relación mantenida entre los diferentes movimientos sociales y Morales. El resultado de ambos cambios no provocó un impacto homogéneo en la compleja realidad societaria boliviana. En ese sentido, desde los inicios de su primera administración, se fue gestando en el seno de diferentes sectores sociales y grupos corporativos una suma de tensiones no zanjadas.

Si bien durante el inicio de su segundo mandato presidencial Evo logró una mayoritaria adhesión a su proyecto, a pesar de ello, hubo sectores empresariales con estrechos vínculos con Estados Unidos y grupos regionales que cuestionaron su esquema de gobierno. Sin duda, el intento reeleccionista que se desarrolló en toda su tercera gestión sumado al desconocimiento de la derrota comicial en el referéndum de 2016 hizo que los cuestionamientos de esos grupos comenzasen cada vez más tener un mayor eco sobre diversos ámbitos sociales. 


\section{La politica primario-exportadora del MAS}

De acuerdo con el exministro de economía Luis Arce (2011) el modelo económico del MAS se sustentó sobre la base de dos pilares: un sector estratégico que generaba excedentes a través de la explotación de hidrocarburos, minería, electricidad y los recursos ambientales junto con otra esfera que creaba ingresos y empleo en el ámbito de la industria manufacturera, turismo, vivienda, etc.; en donde el Estado jugaba un papel central para transferir los recursos de los sectores excedentarios a los segundos. A pesar de que el objetivo final que se propuso fue el de erigir un país industrial, se puede concluir que tras trece años de administración no hubo una modificación significativa del sistema primario exportador. La estructura económica permaneció encadenada a una lógica extractivista nutrida por los altos precios mundiales de algunas materias primas, donde el endeudamiento externo constituyó una pieza central en su funcionamiento.

Como producto de esta orientación económica, Bolivia alcanzó una tasa promedio de 5,1\% de crecimiento del Producto Interno Bruto (PIB) entre 2006 y 2014, a pesar de la crisis mundial del 2009 (Ministerio de Planificación del Desarrollo, MPD, 2016, p. 48). Estas cifras, en gran medida, se explican por el significativo incremento de las exportaciones de materias primas dentro de un ciclo de alza en los precios internacionales de los commodities; sobre todo, por los productos provenientes de las ramas de hidrocarburos y de mineria.

De esta manera, la producción primaria de bienes no renovables siguió teniendo un peso central en la estructura económica tal como lo formuló el Plan de Desarrollo Económico y Social 2016 - 2020. Según el Instituto Nacional de Estadística (INE, 2014), estas actividades representaron el 66,9\% de las exportaciones: hidrocarburos $(51,2 \%)$ y minerales $(15,8 \%)$; por su parte, las operaciones vinculadas al agro y a la industria manufacturera intervinieron tan solo con el $33,1 \%$ de las exportaciones (MPD, 2016, p. 24). Por otra parte, bajo las presidencias del MAS las ventas al exterior de textiles, prendas de vestir, joyería de oro, muebles de madera, etc., cayeron abruptamente en términos absolutos frente a la participación dominante de los envíos de gas natural.

En correspondencia con ello, el gobierno durante los primeros años disfrutó de una balanza comercial con superávit; sin embargo, este panorama cambió en el último lustro. A partir del primer trimestre de 2015 se registró una caída abrupta, lo que provocó un importante saldo deficitario. Según el Instituto Boliviano de Comercio

\section{anuario.}


Exterior (IBCE) esto se produjo debido al derrumbe del precio internacional de los hidrocarburos (sobre todo el petróleo) y los minerales. A eso se sumó el descenso de las ventas a los principales socios comerciales: Brasil, Argentina, Estados Unidos.

Todo este esquema descansó en la prioridad otorgada a la explotación de bienes primarios. Esto se expresó tanto en la inversión privada como en las políticas públicas dirigidas hacia las ramas extractivas. Un caso que ejemplifica esta situación fue el fuerte vínculo que se estableció con China a partir de la visita de Morales en el 2011. Desde ese momento, en el marco de lo que el gobierno consideró a esta relación como una "asociación estratégica", se suscribieron dieciséis acuerdos en materia de comunicaciones, seguridad alimenticia, energía, minería y transporte. De esa manera, las firmas asiáticas se convirtieron en las mayores contratistas de estas inversiones, beneficiándose con numerosas licitaciones y adjudicaciones directas. Además, esto se encuadró dentro de un nuevo esquema de contrataciones públicas, donde se cedió la estimación de los presupuestos y el posible costo de las inversiones a las compañías ejecutoras de los proyectos, desistiendo el Estado su papel de regulador del capital (Centro de Estudios para el Desarrollo Laboral y Agrario, CEDLA, 2018).

Como se mencionó, la extracción de bienes minerales no renovables siguió teniendo un peso central en la estructura económica. Si bien Evo en la campaña electoral de 2005 prometió un renacimiento de la minería estatal, sus administraciones se caracterizaron por fortalecer al ámbito privado representado tanto por las cooperativas mineras como por la mediana y gran minería en manos de las transnacionales. De este modo, se produjo una expansión de la escala de esa actividad tanto en la extensión de los territorios explotados como en el incremento en la cantidad y variedad de los productos minerales exportados.

Según el Ministerio de Minería y Metalurgia (MMyM, 2013) la participación estatal en la producción global minera fue de $7,85 \%$ frente al ámbito privado que superó el $92 \%$. En este sentido, es importante mencionar que los procesos de estatización se sustanciaron en escasas oportunidades; en la mayoria de las ocasiones, estos se alcanzaron tras una serie de enfrentamientos entre asalariados mineros, cooperativistas (socios y operarios) y comunitarios originarios (Schneider, 2016). O bien, la participación en algunos casos fue en sociedad con empresas privadas, como fue el proceso que habilitó la industrialización del litio a partir de la creación de la empresa mixta de Yacimientos de Litio Bolivianos (YLB) junto con la alemana ACI Systems, transnacional que iba a controlar el $49 \%$ del desarrollo manufacturero. ${ }^{1}$ Por su parte, el sector privado fue el principal actor en la

1 Cabe observar que entre los grupos que se movilizaron contra Morales en noviembre de 2019 se encontraba un importante sector de la población del departamento de Potosí que se oponía a la 
Tensiones económicas, políticas y sociales durante los gobiernos de Evo Morales. Una interpretación sobre el golpe de Estado

comercialización de productos con el exterior; así, entre otros productos, las cooperativas concentraron el $91 \%$ de la venta de oro, mientras que las multinacionales dominaron la producción de zinc, plata y plomo controlando los yacimientos de San Cristóbal, San Bartolomé y San Vicente, siendo responsables del 52\% del total de las exportaciones entre el 2006-2012 (MMyM, 2013; Díaz, 2017 , p. 46). ${ }^{2}$

Al igual que en la exportación de minerales e hidrocarburos, las presidencias del MAS han favorecido la producción de materias primas agrícolas a fin de satisfacer la demanda del mercado externo. En todos los casos se privilegió la defensa de la propiedad de la tierra, incluso la de los grandes empresarios privados. Además, se concedieron inversiones en infraestructura productiva $\mathrm{y}$ transporte, precios rentables y se subsidió a un precio bajo el valor del diésel como combustible para el sector rural. Al calor del incremento mundial de los precios de algunos productos agrícolas (como la soya, el girasol, la caña de azúcar), el agronegocio se extendió como forma de desarrollo de la producción agropecuaria en concordancia con una mayor extranjerización de la propiedad, en particular, en Santa Cruz donde creció la presencia de empresarios agrícolas ganaderos de Brasil y Argentina (Urioste, 2011).

Como parte de este esquema, cuyo eje central giró en torno al fortalecimiento de los sectores agroindustriales de los departamentos orientales del país, se amplió la frontera agrícola por medio de diversas campañas de deforestación. Las mismas fueron impulsadas a través de proyectos estatales y privados con construcciones de carreteras, concesiones para la explotación de hidrocarburos, etc. Desde el 2013, el gobierno aprobó varias leyes, decretos y normas técnicas que dieron lugar a la deforestación; estas medidas de avance territorial se ampliaron con la ley 741 de 2015 por la que se facultó los desmontes de hasta veinte hectáreas y el decreto supremo 3973 de 2019 que autorizó las quemas en los departamentos de Santa Cruz y Beni. ${ }^{3}$ La explotación de la tierra y de los bosques no solo han favorecido a grupos agroexportadores y a las petroleras transnacionales sino también a empresarios agrícolas y ganaderos, a grandes explotadores de madera y a pequeños propietarios (Wanderley et.al., 2018). En otras palabras, se ha desarrollado un constante flujo poblador hacia a esa zona por medio de colonizadores, cocaleros,

aprobación del decreto supremo que permitía el acuerdo con la firma alemana para la industrialización del litio. Ante ese reclamo, pocos dias antes de que Evo renuncie, el decreto fue derogado.

2 Durante 2013 la exportación de minerales de las cooperativas fue tres veces mayor a la del sector estatal. Por otro lado, las empresas públicas no participaron de la explotación de minerales como plomo, antimonio y wólfram (Página Siete, 13 de abril de 2014). En un sentido similar, en el 2018 las cooperativas auríferas exportaron 1.165 millones de dólares; sin embargo, erogaron por regalías tan solo el 2,5\%, es decir, unos 30 millones de dólares (Página Siete, 31 de enero de 2019).

3 Valga subrayar que esta concesión provocó el devastador incendio en el Bosque Seco Chiquitano en agosto y septiembre del año 2019.

\section{anuario.}


entre otros grupos, perjudicando a los colectivos indígenas

tradicionales de las tierras bajas, con un significativo impacto a nivel social y ambiental, tal como se observa con lo sucedido por el avance de esa política económica en el Territorio Indígena Parque Nacional Isiboro Sécure (TIPNIS).

\section{Limites y alcances de la politica distributiva del gobierno}

De acuerdo con Arce (2011) el modelo económico ha sido exitoso durante todos estos años por la activa intervención del Estado; en particular, gracias a la nacionalización de los hidrocarburos. Ahora bien, al respecto es válido subrayar que esta medida fue notoriamente diferente a las que se efectuaron durante el siglo XX. ${ }^{4}$ A pesar del discurso propagandista del gobierno alrededor de una épica nacionalista, esta norma no implicó la expulsión de las empresas privadas del sector sino más bien su permanencia bajo nuevas condiciones impositivas, sin impugnar su presencia hegemónica. ${ }^{5}$

En esa coyuntura, se orientó hacia un incremento del gravamen cobrado a las empresas transnacionales beneficiadas por el ciclo alcista de los precios, sin incidir tanto en el manejo real de la extracción como en la cantidad de utilidades y ganancias que las firmas giran a sus respectivas casas matrices en el exterior. De ese modo, la ley apuntó tan solo a reformar el régimen tributario con el fin de mejorar la percepción fiscal. En ese esquema, cabe observar que no se dio un auténtico proceso de incautación porque la fase central del proceso de producción lo siguieron controlando las compañias extranjeras que continuaron siendo las principales responsables de la producción de gas natural y de hidrocarburos líquidos; de ahí se comprende por qué ninguna firma privada se haya retirado de este negocio (Gandarillas, 2016).

La consecuencia inmediata de la denominada nacionalización de los hidrocarburos y de algunas empresas mineras (por ejemplo, Huanuni) derivó en una significativa recaudación impositiva, lo que permitió al gobierno aplicar tanto una política de ampliación del consumo doméstico como de mayor gasto estatal. En particular, una parte de la renta proveniente de las exportaciones se dirigieron al pago de un incentivo a la permanencia escolar (el Bono Juancito Pinto), la Renta Dignidad para los ancianos y el Bono Juana Azurduy para mujeres gestantes que no cuentan con un seguro de salud; logrando una mejora sustancial en el nivel de vida de amplios sectores de la población. De idéntica manera, con lo obtenido por esos medios

\footnotetext{
4 A diferencia de la nacionalización de Morales, en los anteriores procesos (1937 y 1969) se produjo una radical confiscación de los bienes de las empresas extranjeras.

5 La medida fue dictada el primero de mayo de 2006 a través del decreto "Héroes del Chaco", en referencia a los bolivianos que fallecieron en la guerra contra el Paraguay (1932 -1935).
}

\section{anuario.}


Tensiones económicas, políticas y sociales durante los gobiernos de Evo Morales. Una interpretación sobre el golpe de Estado

sumado a otras fuentes de financiamiento, se impulsaron diversos programas de construcción pública como el denominado "Bolivia Cambia, Evo cumple", dirigido a sostener una serie de proyectos municipales en el ámbito de la salud, educación, desarrollo productivo e infraestructura social.

Este panorama económico tuvo su correlato en el ámbito de la ocupación. Durante las presidencias del MAS, en términos generales, aumentó el empleo. De acuerdo con el esquema concebido en el Plan Nacional de Desarrollo (Ministerio de Economía y Finanzas Públicas, MEFP, 2006), el incremento debía producirse a partir del impulso dado por el Estado a las pequeñas y medianas unidades productivas de bienes y servicios. Al respecto, como producto de esta orientación económica, la tasa de desempleo abierto urbano disminuyó de 8,1\% en el 2005 a 4,5\% en el 2017 (MEFP, 2018); en consonancia con el modelo productivo el aumento se dio en aquellos establecimientos que empleaban escaso personal en el lugar. ${ }^{6}$

Sin embargo, este incremento ocupacional no fue uniforme ya que en el citado período se sucedieron claramente dos fases: la primera, entre el 2006 y el 2012, hubo un prolongado crecimiento económico debido a la mejora de los precios de las materias primas en los mercados internacionales; en tanto, en un segundo momento, por circunstancias opuestas a las anteriores, se produjo un proceso de desaceleración entre 2013 y 2015. Ahora bien, en correspondencia con las características del modelo primario exportador, el empleo se encontró agrupado sobre todo en los rubros de comercio y servicios.

Por otro lado, una de las características nodales de la economía boliviana es el continuo crecimiento del empleo informal. Según el INE, en el 2014, el 60\% de la población ocupada en el ámbito urbano se hallaba trabajando en actividades de este tipo; como consecuencia de la mencionada desaceleración esta tendencia fue en aumento, alcanzando al $67 \%$ de los ocupados en el último trimestre de 2017.7 De esa manera, la mayoría de las unidades productivas del área de la construcción, del comercio, los servicios y de las faenas agropecuarias de subsistencia se caracterizaron por un exiguo nivel de productividad, un bajo desarrollo tecnológico y una débil organización institucional.8 Si bien a partir de 2011 el trabajo por cuenta propia (sobre todo, en la construcción, los servicios personales y el transporte público) proporcionó un aumento de la ocupación, esto condujo a un incremento de la precariedad laboral en esas ramas, con prácticas de

\footnotetext{
6 En el ámbito urbano nacional, el 90,1\% de las unidades económicas tiene menos de cinco personas ocupadas, mientras que, en el otro extremo, las empresas con 50 y más ocupados apenas conforman el 0,4\% (Ministerio de Trabajo, Empleo y Previsión Social, 2017).

${ }^{7}$ Al respecto véase, entre otros, (Página Siete, 28 de abril de 2019).

8 Cabe indicar que los empleos precarios carecen de convenio laboral a la vez que no poseen ni jubilación ni indemnización por despido.
}

\section{anuario.}


subcontratación y tercerización. El 65\% de la ocupación y el empleo

urbano se concentraron en los sectores familiares y en las pequeñas empresas, con relaciones salariales que se hallan al margen de los derechos laborales (Escóbar, Arteaga, Hurtado, 2019, p. 50). En ese sentido, el porcentaje de trabajadores que tienen una ocupación estable, bien remunerada y protegida por la seguridad social se redujo en la última década a solo 20,9\%; el resto mantuvo un empleo precario (79,1\%) (CEDLA, 2013). En un sentido similar, Ernesto Yáñez (2018), sobre la base de cifras oficiales, subrayó que entre 2006 y 2015 la calidad del empleo no mejoró pese al prolongado período de crecimiento económico. ${ }^{9}$

En cuanto a las medidas de aumento salarial cabe observar que el Poder Ejecutivo adoptó un criterio fiscalista a la hora de decidir el porcentaje de incremento en los haberes. Por lo general, el monto en que se calculó la suba de estos estuvo sujeta a la tasa inflacionaria del año anterior, lo que no significó que el incremento se corresponda con la real capacidad de compra de bienes y servicios. Asimismo, los reajustes salariales se restringieron solo a los trabajadores que recibian un monto salarial, igual o inferior al mínimo nacional; en cambio, en los restantes sectores de la población los haberes se fijaron por el diferente régimen de contratación, ya fuesen permanentes o eventuales. Por otro lado, no menos importante es que, al igual de lo que sucede en materia de empleo, la discriminación por género también se manifestó en el hecho de que por idénticas tareas las mujeres percibieron un salario hasta un 30\% menor que los hombres (INE, 2018).

En otros temas, durante el último año de la presidencia de Morales hubo una intensa discusión entre sectores oficiales y organismos privados (nacionales y extranjeros) sobre el nivel de pobreza. De ese modo, según el gobierno tanto la pobreza extrema como la moderada se redujeron a cerca de la mitad entre 2005 y 2018 (MEFP, 2018). Sin embargo, desde otra perspectiva, el CEDLA argumentó que las cifras serian superiores; ya que el $61 \%$ de los bolivianos se encontrarian bajo ese escenario (Escóbar, Arteaga, Hurtado, 2019). ${ }^{10}$

\footnotetext{
9 Cabe indicar que esta investigación consideró el índice de la calidad del empleo, sobre la base de tres dimensiones: ingreso, satisfacción y estabilidad laboral; la misma se hizo a partir de datos oficiales proporcionados por el INE.

10 Sobre las divergencias en las mediciones entre los distintos organismos se puede consultar (Página Siete, 12 de marzo y 1 de agosto de 2019). Asimismo, habría que subrayar que el CEDLA empleó un criterio más amplio de medición: el llamado Índice de Pobreza Multidimensional (IPM) en el cual se consideran otras variables a la hora de extraer conclusiones.
} 
Tensiones económicas, políticas y sociales durante los gobiernos de Evo Morales. Una interpretación sobre el golpe de Estado

Los vínculos entre los movimientos sociales y el gobierno del MAS

El extenso ciclo gubernamental de Morales ha transitado por distintos momentos de aceptación o rechazo en el transcurso de sus trece años de gestión. Así, desde haber obtenido un alto nivel de aprobación en las elecciones presidenciales de 2009 y 2014 pasó a la abrupta deriva que finalizó con su renuncia el pasado 10 de noviembre. En gran medida, este versátil escenario se explica observando una serie de tensiones y contradicciones que se fueron gestando entre su administración y las distintas organizaciones sociales que fueron su principal base de apoyo durante su primera presidencia cuando enfrentó a los prefectos de los departamentos del oriente boliviano. De este modo, en esta sección, se examinarán los complejos y cambiantes vínculos entre algunas organizaciones sociales y el Poder Ejecutivo; en particular, se estudiará tanto el papel de algunos pueblos indígenas como el oscilante comportamiento de la dirigencia de la Central Obrera Boliviana (COB).

La primera presidencia estuvo signada centralmente por los profundos conflictos que protagonizó el gobierno contra las autoridades de Santa Cruz, Tarija, Beni y Pando en el marco de los debates de la Asamblea Constituyente. Cuando estos enfrentamientos se agravaron, el MAS recurrió a la movilización y a la acción callejera de amplios sectores sociales apelando para ello a una estructura organizacional creada en el 2004: el Pacto de Unidad. Esta entidad se hallaba constituida por una serie de organizaciones campesinas e indígenas: la Confederación Sindical Única de Trabajadores Campesinos de Bolivia (CSUTCB), la Confederación Sindical de Colonizadores de Bolivia (CSCB), la Confederación Nacional de Mujeres Campesinas Indígenas Originarias de Bolivia Bartolina Sisa (CNMCIOB-BS), la Confederación de Pueblos Indígenas del Oriente Boliviano (CIDOB), el Consejo Nacional de Ayllus y Markas del Qullasuyu (CONAMAC), la Coordinadora de Pueblos Étnicos de Santa Cruz (CPESC), el Movimiento de Trabajadores Campesinos Sin Tierra de Bolivia (MST-B), la Asamblea del Pueblo Guaraní (APG), la Central de Pueblos Étnicos Mojeños del Beni (CPEMB) y la Asociación Nacional de Regantes y Sistemas Comunitarios de Agua Potable y Saneamiento (ANARESCAPYS).

A pesar de las diferencias internas que mantenian esas organizaciones, las mismas compartieron una serie de agendas similares relacionadas con demandas de reconocimiento étnico, tierras y/o territorio e inclusión política, lo que explica -en parte- el interés de efectuar sendos cambios en la estructura legal del país. En ese contexto, en la coyuntura de oposición con las fuerzas cívico-regionales del oriente boliviano decidieron sostener al partido oficial; como consecuencia, en enero de 2007, se formalizó la Coordinadora Nacional para el Cambio (CONALCAM). De ese modo, desde sus orígenes, esta se proyectó como una instancia de coordinación integrada por representantes de las diferentes organizaciones matrices junto con

\section{anuario.}


integrantes del Poder Ejecutivo, del legislativo y representantes de la convención constituyente respaldando el denominado Proceso de Cambio liderado por Morales. En la práctica, en los momentos más tensos durante los enfrentamientos de mediados de 2008, la CONALCAM se convirtió en el principal instrumento que empleó el gobierno para contrarrestar en las calles y en las rutas el desafio de los prefectos de los departamentos mencionados. Más aún, tras la masacre en la localidad de El Porvenir (Pando), el presidente logró incorporar a la dirigencia de la $\mathrm{COB}$ a la coordinadora, reforzando los lazos entre las organizaciones sociales y el MAS. Sin embargo, cabe subrayar que la incorporación de la central obrera generó numerosas críticas en el seno de sus organismos internos; sobre todo, porque se produjo pocas semanas después de una brutal represión policial sobre un contingente de mineros asalariados que se encontraban bloqueando caminos en el cruce de Caihuasi. ${ }^{11}$

Luego de ser aprobada la Constitución Política del Estado en el 2009, la CONALCAM terminó de alinearse con el MAS a través de diferentes oficinas gubernamentales, consolidando una fuerte instancia clientelar y corporativa. En forma temprana, Zuazo (2010) observó que la sanción de la Carta Magna habilitó la apertura de ciertos mecanismos legales para incorporar los movimientos sociales al Estado. Al respecto, uno de los instrumentos que el gobierno utilizó y que tuvo una amplia repercusión durante esos años fue el empleo de dinero por parte de algunos dirigentes sectoriales a través del Fondo de Desarrollo para los Pueblos Indígenas, Originarios y Comunidades Campesinas (FDPPIOYCC). Si bien en un principio el organismo se creó con el mandato de financiar proyectos de desarrollo productivo y social para beneficiar a los sectores antes mencionados, en noviembre de 2014 emergieron una serie de denuncias de corrupción que involucraron tanto a altos dirigentes de las Bartolinas, de la CSUTCB y de los Interculturales (ex colonizadores) como a funcionarios públicos (Morales, 2015).

A pesar de ello, la cooptación no significó un proceso sencillo. Durante los siguientes años, el primer mandatario tuvo serias dificultades para mantener la cohesión y la adhesión al partido oficial de aquellas organizaciones que integraron en sus origenes la CONALCAM. En el transcurso de sus presidencias, algunas centrales se alejaron (CONAMAQ, CIDOB, etc.) y otras mantuvieron una actitud oscilante como fue el caso de la COB. Sin embargo, el gobierno no se resignó. En numerosas oportunidades, declaró la necesidad de que la CONALCAM asumiera como tarea nodal el adoctrinamiento político y la preservación del Proceso de

11 El alineamiento con el MAS generó una fuerte crisis en el sindicato de trabajadores mineros de Huanuni, lo que derivó en la renuncia de algunos de sus dirigentes. En idéntico sentido, las centrales obreras departamentales de Oruro y Tarija cuestionaron a la dirección de la COB encabezada por Pedro Montes por la subordinación a los intereses el primer mandatario, quebrando con la tradición de independencia de clase que debería tener el órgano matriz de los asalariados. 
Tensiones económicas, políticas y sociales durante los gobiernos de Evo Morales. Una interpretación sobre el golpe de Estado

Cambio; a la vez, abogó para que se convierta en un "Consejo de Estado".12 En idéntico sentido, en consonancia con el alineamiento, en no pocas ocasiones, algunas de esas entidades se enfrentaron verbal y físicamente con los sectores sociales que hacían medidas de fuerza contra el gobierno (Quiroga et.al, 2012; Schneider, 2018).

Por otro lado, por fuera de los movimientos sociales hubo distintos grupos empresariales y fuerzas cívico-regionales del oriente boliviano que, si bien se relegaron parcialmente del escenario politico aceptando el nuevo esquema de fuerza que se impuso tras la sanción de la constitución y de los resultados comiciales de diciembre de 2009 y de 2014, siempre fueron opositores. Prueba de ello fue la tenaz y sistemática impugnación que llevó a cabo el expresidente Jorge "Tuto" Quiroga y sus seguidores. De ese modo, en reiteradas ocasiones calificó al gobierno como fraudulento ya que este se habría "aprovechado de los movimientos sociales, pervirtiéndolos y convirtiéndolos" en "corporaciones mafiosas". ${ }^{13}$ La coyuntura abierta con el desconocimiento del resultado de los comicios de febrero de 2016 por Morales junto con su reintento de una tercera reelección hizo que las declaraciones del dirigente conservador cobraran mayor fuerza y eco en diferentes sectores de la población que cuestionaban (aunque por diversos motivos) la gestión de Evo. En forma paralela, la negligencia gubernamental condujo a que los sectores históricos de la oposición empezaran a articularse tras la bandera de la defensa de la democracia y el veto a una nueva candidatura presidencial del mandatario campesino; fenómeno que se canalizó primero con el Comité Nacional en Defensa de la Democracia (CONADE) y luego, en gran medida, con la candidatura del expresidente Carlos Mesa.

\section{El avance de las politicas extractivistas: el caso del TIPNIS}

Uno de los acontecimientos centrales donde se evidenciaron tanto las tensiones con el gobierno como en el seno de la CONALCAM fue durante los conflictos suscitados alrededor del avance de las medidas de corte extractivista que se dieron en el área del TIPNIS. ${ }^{14}$ Como se ha mencionado, una de las primeras acciones de Morales bajo su segundo mandato, en el marco de su Plan Nacional de Desarrollo (MEFP, 2006), fue la de impulsar una serie de proyectos regionales de construcción de

\footnotetext{
12 Para una mayor comprensión de las tareas propuestas, véase (La Razón, 29 de enero de 2013 y La Razón, 17 de abril de 2018).

13 Sobre el particular, véase, (Página Siete, 18 de febrero de 2016).

$14 \mathrm{El}$ área fue creada en 1965 como parque nacional debido a su amplia diversidad biológica y por su enorme riqueza hídrica. En 1990, como resultado de la Marcha por el Territorio y la Dignidad de los pueblos indigenas de tierras bajas, la zona fue reconocida como territorio ancestral de los pueblos mojeño, yuracaré y chimán (Cortez, 2011).
}

\section{anuario.}


transporte, energía y telecomunicaciones en los departamentos orientales. Dentro de estas iniciativas se encontraba la idea de construir una autopista bioceánica. Si bien esta era una aspiración de larga data, en el 2010, el presidente firmó un crédito con el Banco Nacional de Desarrollo Económico y Social de Brasil para financiar esa inversión en el marco de la Iniciativa para la Integración de la Infraestructura Regional Suramericana (IIRSA) (Fortún, 2012). En este sentido, cabe indicar que detrás de este megaproyecto se encontraban intereses sectoriales tanto de capitales nativos como extranjeros involucrados en la exploración de hidrocarburos, en la producción agropecuaria, en el cultivo de coca, entre otros bienes para la exportación (Quiroga et al., 2012).

En ese escenario, la principal forma en que se manifestó esta pugna fue a través de las marchas opositoras a La Paz en contra de la construcción del proyecto carretero que pretendió pasar por ese territorio intangible. Las demandas fueron encabezadas por la CIDOB quienes centralmente agruparon los reclamos de los pueblos Mojeño, Yuracaré y Chimán. Además, no solo buscaron detener esa obra, sino que también solicitaron la titulación colectiva de tierras, la gestión territorial indígena autónoma, el empleo y el aprovechamiento exclusivo de los recursos naturales renovables existentes, la consulta a través de sus instituciones y la creación de una universidad indigena.

En esa coyuntura, la movilización más importante fue la VIII Marcha Indígena por la Defensa del TIPNIS y la Dignidad de los Pueblos Indígenas de La Amazonía, Oriente y Chaco efectuada entre los meses de agosto y octubre de 2011. La marcha encabezada por la CIDOB fue respaldada por la CONAMAQ, la APG, la CPEMB, la Central de Pueblo Indígenas del Beni (CPIB), la Central Indígena de Pueblos Originarios de la Amazonía de Pando (CIPOAP), la Central de Mujeres Indígenas del Beni (CMIB), la Central Indígena de la Región Amazónica de Bolivia (CIRABO), la Central de Pueblos Indígena de La Paz (CPILAP), la Coordinadora de Pueblos Indígenas del Trópico de Cochabamba (CPITCO), la Organización Indígena Chiquitana $(\mathrm{OICH})$ y la Confederación Nacional de Mujeres Indígenas de Bolivia (CNAMIB). A medida que fueron pasando las semanas, la medida fue sumando un amplio apoyo de la población (entre otros de la COB), sobre todo, tras una serie de incidentes con el gobierno y con organizaciones afines al mismo como los colonizadores. ${ }^{15}$ Tras esos acontecimientos, luego de recorrer cerca de seiscientos cincuenta kilómetros, la llegada a La Paz fue recibida calurosamente por distintos sectores de la ciudadanía que se volcaron a las calles para expresar su apoyo a los indigenas.

15 Los acontecimientos más graves ocurrieron con el bloqueo a los marchistas protagonizado por los colonizadores interculturales en Yucumo junto la represión policial con heridos en la localidad de Chaparina. 
Tensiones económicas, políticas y sociales durante los gobiernos de Evo Morales. Una interpretación sobre el golpe de Estado

Frente a ello, el primer mandatario promulgó la Ley $\mathrm{N}^{\circ} 180$ de intangibilidad del TIPNIS. Sin embargo, la concesión fue momentánea. A comienzos del 2012, mientras impulsaba la creación de una organización de campesinos cocaleros cercana a su gobierno, el denominado Consejo Indígena del Sur (CONISUR), Morales promulgó la Ley $\mathrm{N}^{\circ} 222$ por la que convocó a una consulta previa para la construcción de la carretera. Ante ello, los originarios de la CIDOB efectuaron una IX Marcha Indígena por la Defensa de la Vida y la Dignidad, los Territorios Indígenas, los Recursos Naturales, la Biodiversidad, el Medio Ambiente y las Áreas Protegidas en el mes de abril de ese año, a la vez que decidieron rechazar el cuestionario desde las propias comunidades del lugar. A pesar de esos enfrentamientos que le generaron un severo desgaste a nivel nacional e internacional, Evo decidió seguir con la construcción de la autopista en 2017, luego de un breve período de suspensión del tema. De ese modo, a través de la promulgación de la Ley 969 anuló la Ley 180 que le otorgaba al TIPNIS la intangibilidad desde el 2011.16

De manera clara y contundente en las protestas de los pueblos indigenas de las tierras bajas salió a la luz una de las principales tensiones inherentes al denominado Proceso de Cambio: la lógica que privilegia el desarrollo de un capitalismo industrial a partir de la integración al mercado mundial frente a otra que sostiene la defensa de determinados valores ecológicos, sociales y comunitarios. Los originarios de la zona argumentaron en todo momento su derecho de preservar y decidir sobre sus propios modos de vida, la defensa de sus territorios ancestrales, la protección de los bosques y las aguas y su oposición al avance de nuevos actores económicos y sociales como los cocaleros, los ganaderos, las empresas de hidrocarburos, etc. Por el contrario, en numerosas ocasiones, Álvaro García Linera $(2011,2012)$ se encargó de defender esas medidas justificando el avance sobre esa región con el objetivo de fortalecer el modelo de exportación de bienes primarios, sobre la base de conformar un "capitalismo andino amazónico" (2006).

El enfrentamiento por el control del TIPNIS se convirtió en un hecho emblemático para la presidencia de Morales debido a que diversas leyes nacionales reconocen su doble condición: como parque nacional y como territorio indígena. Además, la Constitución del 2009 estableció para los pueblos indígenas la preexistencia de sus naciones, su autogobierno, el manejo de los recursos naturales y el derecho a la consulta. Por otra parte, como resultado de estas medidas y como consecuencia de la represión policial durante la marcha de septiembre de 2011 se produjo el resquebrajamiento de la CONALCAM: de ese modo se alejaron la CIDOB y la CONAMAQ; en tanto quedaron alineadas con el MAS la CSUTCB, los colonizadores y la CNMCIOB-BS que exigieron la construcción de la autopista.

16 Sobre el particular, véase, (Página Siete, 24 de agosto de 2017). 
Esta no fue la única ruptura con el gobierno. En forma simultánea, un grupo de intelectuales, exfuncionarios y distintos dirigentes sociales locales se alejaron del MAS a partir de una serie de críticas en materia económica, política y ambiental, esbozando la necesidad de reconducir el Proceso de Cambio (VV.AA, 2012). Frente a este documento firmado por Alejandro Almaraz, Raúl Prada, entre otros, García Linera (2011) escribió un libro en donde se descalificó en forma despectiva las críticas de sus antiguos compañeros de ruta. ${ }^{17}$

\section{Una central sindical cada vez más integrada al gobierno}

En ese marco es interesante observar, a grandes rasgos, cómo fue cambiando el vínculo entre la dirigencia de la $\mathrm{COB}$ y el gobierno. En los inicios del mandato presidencial, los líderes laborales sostuvieron una actitud de desconfianza hacia Morales: le cuestionaron dos grandes temas. En primer lugar, se criticó que el ministerio de minería estuviese encabezado por un empresario cooperativista, quebrando con la tradición de que tenía que ser un trabajador minero. En segundo término, se esbozó la preocupación de que las organizaciones sociales ligadas a Evo buscasen modificar el estatuto de la entidad e impusieran a dirigentes de sindicatos campesinos al frente de la misma en el XIV Congreso Orgánico de junio de 2006. Sin embargo, esto último no sucedió ya que los delegados al mitin hicieron prevalecer la tradición minera a la hora de elegir una nueva conducción. De esta forma, el encuentro designó a Pedro Montes en reemplazo de Jaime Solares como Secretario Ejecutivo. A la par de esta nominación, el cónclave reafirmó "la nacionalización sin indemnización de los hidrocarburos, la recuperación de todos los recursos naturales y la abrogación de los decretos y leyes neoliberales" (CEDLA, 2009, p. 5).

A pesar de estas declaraciones, durante los siguientes años la dirigencia encabezada por Montes se terminó acercando al proyecto del Poder Ejecutivo como se evidenció con el ingreso de la central obrera a la CONALCAM. Con excepción del conflicto ocurrido en Huanuni (2006) y de las discusiones por la modificación de la Ley de Pensiones (2008), se produjo una explícita convivencia entre ambos actores a lo largo del primer mandato del MAS. ${ }^{18}$

\footnotetext{
17 Las criticas y las censuras contra intelectuales y Organizaciones No Gubernamentales (ONG) no se detuvieron. Durante el segundo semestre de 2015 hubo un serio intento de expulsar a dos de estas entidades y a dos fundaciones por las criticas que efectuaban al gobierno en materia de su politica extractivista (Página Siete, 11 de agosto de 2015). Durante los años siguientes, 2016 y 2017, la ofensiva desde el Poder Ejecutivo continuó a través de la censura, el congelamiento de sus cuentas bancarias, etc. (Página Siete, 24 de septiembre y 17 de diciembre de 2017).

18 En octubre de 2006 se produjo un sangriento enfrentamiento (en el que fallecieron dieciséis pobladores) entre los socios de las cooperativas con los asalariados mineros por el control del cerro Posokoni. A raíz de ello, Morales firmó un decreto para nacionalizar el yacimiento; asimismo, desplazó
} 
Tensiones económicas, políticas y sociales durante los gobiernos de Evo Morales. Una interpretación sobre el golpe de Estado

Sin embargo, este escenario cambió durante el segundo período presidencial. A partir del triunfo electoral del 2009, el gobierno decidió impulsar con fuerza su proyecto económico, lo que derivó en un incremento de las protestas sociales; en particular, las encabezadas por la clase trabajadora (Fundación UNIR, 2012). En función de ello, creemos que es importante detenerse a subrayar la conflictividad ocurrida en esos años ya que la misma resulta de amplia utilidad para observar las tensiones entre unas bases sociales que poseen una agenda propia y distinta a la de la dirigencia sindical que se encuentra alineada con el gobierno.

Cabe indicar que el recrudecimiento de la protesta se dio en la mayoria de las ocasiones por fuera de la voluntad de la cúpula. De esa manera, las primeras medidas de 2010 fueron efectuadas por los docentes y el proletariado fabril en oposición al anteproyecto del Código de Trabajo que era impulsando por el oficialismo en connivencia con la dirigencia laboral. ${ }^{19}$ Este panorama se intensificó al cruzarse con las negociaciones anuales salariales impulsadas por la central obrera; en esa coyuntura, frente a las diversas críticas que recibía la COB por su actitud diletante, la matriz sindical decidió convocar al primer paro general nacional del periodo. Este tuvo un amplio apoyo con marchas, bloqueos de vías y piquetes de huelga de hambre; debido a esa masiva demostración de fuerza Montes decidió acordar el aumento dispuesto originalmente por el presidente. En ese escenario solo quedaron protestando, en forma aislada durante un par de semanas, los docentes urbanos y los obreros fabriles de La Paz. ${ }^{20}$

Pese a que la COB había intentado cerrar el 2010 con calma, esta meta no se pudo lograr. Un nuevo motivo de disputa se inició a fines de diciembre a partir del decreto de García Linera de eliminar los subsidios a los combustibles. A raíz de este hecho, se produjo una veloz suba de los precios, lo que derivó en una protesta masiva de toda la población. En pocas horas, mineros, docentes, juntas vecinales, transportistas, seccionales de sindicatos fabriles y de amas de casa exigieron la derogación del gravamen junto con la renuncia de varios funcionarios, si no se retrocedía con la medida. Al igual que en otras ocasiones, las medidas de fuerza se desplegaron por fuera de la voluntad de la cúpula de la central sindical, que fue duramente increpada por sus bases por la actitud dubitativa. Tras una semana de paro, a pocas horas de que finalice el año, Morales se vio obligado a derogar el decreto.

a su ministro de Minería que respondía a los intereses de los empresarios cooperativistas (Schneider, 2016).

19 El cuestionamiento al anteproyecto se centró en que se limitaba el derecho de huelga, reglamentando los pasos a seguir antes y después de declarada la medida de fuerza.

20 Véase, (La Prensa y La Razón, mayo de 2010).

\section{anuario.}


Durante el 2011 las protestas se originaron en reclamo de aumentos

salariales, frente a la suba importante que hubo de precios en los productos de primera necesidad y del transporte. De esa manera hubo paros nacionales y parciales de medio centenar de asociaciones sindicales, federaciones y centrales obreras departamentales y regionales, con bloqueos de caminos y marchas. Tampoco estuvo ausente la represión policial contra mineros y maestros rurales con heridos y detenidos. En forma simultánea, el gobierno apeló a sus organizaciones aliadas (campesinos, interculturales, cocaleros) para que se movilizaran en contra de los sectores que protestaban, mientras que Evo y su vice en reiteradas ocasiones descalificaron a los manifestantes como "instrumentos de la derecha", "traidores" y "golpistas" (Schneider, 2018).

Durante el 2012, además de los habituales conflictos por mejoras en los haberes hubo dos grandes pugnas. Por un lado, los médicos protagonizaron una extensa lucha en oposición a la extensión de la jornada laboral de seis a ocho horas en el sistema de salud pública, sin la correspondiente compensación salarial. Luego de cincuenta días de protesta, el Poder Ejecutivo terminó suspendiendo la norma (Leaño, 2012). Por otra parte, los asalariados mineros de Colquiri y Mallku Khota llevaron a cabo intensas protestas contra cooperativistas y empresarios en defensa de la explotación estatal de esos yacimientos. Como resultado de estas luchas, tras cinco meses de medidas de fuerza con paros, enfrentamientos con dinamita (que provocaron decenas de heridos y un muerto), capturas de rehenes, bloqueos de caminos y movilizaciones a La Paz, se consiguió la parcial nacionalización de la primera; en tanto, se logró tras varios años de litigios internacionales que el Estado boliviano se hiciera cargo de la segunda. ${ }^{21}$

De manera simultánea a las protestas mencionadas, hubo conflictos que se originaron por suspensiones, despidos y el cobro de salarios atrasados, ya fuesen de trabajadores estatales (municipales, docentes, empleados de la gobernación de Tarija, trabajadores de la sanidad, etc.) como en el ámbito privado (en el canal Full $T V$, en la alimenticia PIL y en diversas fábricas). Asimismo, en ambos sectores, se desarrollaron numerosas medidas de fuerza en demanda de mejoras de las precarias condiciones de empleo; por ejemplo, en los municipios de Oruro, El Alto y Quillacollo, en la Caja Nacional de Salud, en la Empresa de Correos de Bolivia y en distintas plantas fabriles de La Paz, El Alto, Santa Cruz y Cochabamba.

Con ese panorama de fondo, se acrecentó el cuestionamiento a la dirección cobista de Montes por su permanente alineamiento con el MAS. En ese escenario, la central obrera se reunió en enero 2012 en su XV Congreso Ordinario donde se eligió un nuevo Comité Ejecutivo liderado por el exminero de Huanuni, Juan Carlos Trujillo.

${ }^{21}$ Al respecto, véase, (La Razón y Página Siete, 11 de julio de 2012 y Página Siete, 3 de diciembre de 2018). 
Tensiones económicas, políticas y sociales durante los gobiernos de Evo Morales. Una interpretación sobre el golpe de Estado

Asimismo, el evento declamó y reiteró un conjunto de principios característicos de la historia de la COB centrados en torno al sindicalismo revolucionario. A pesar de que en ese momento se criticó la relación que mantuvo la anterior dirección cobista con Morales, a los pocos meses de iniciada la naciente gestión se reiteraron similares prácticas de colaboración con el gobierno.

En ese escenario, en mayo de 2013 el movimiento obrero protagonizó un importante conflicto; este se originó en torno a la Ley de Pensiones $\mathrm{N}^{\circ} 065$, por la cual el gobierno se aferró a mantener los lineamientos básicos diseñados por el expresidente Gonzalo Sánchez de Losada. A esto se sumaron las protestas ocasionadas por el pedido anual de suba de haberes. Ante ese panorama, la matriz sindical dispuso durante dos semanas cerca de cuarenta puntos de bloqueos en rutas y calles junto con movilizaciones masivas, paro de docentes, médicos, enfermeros, mineros y operarios de fábricas. Entre las acciones más impactantes se puede enumerar el asedio de cuatro mil mineros de Huanuni alrededor de la Plaza Murillo (La Paz) y el bloqueo de trabajadores fabriles y mineros en el cruce de caminos en Caihuasi. Frente a esas medidas de fuerza, el gobierno declaró ilegal la protesta, dispuso la detención de cerca de cuatrocientos trabajadores, reprimió con una decena de heridos de bala en la localidad de Parotani y convocó a sus grupos afines como las Bartolinas a movilizarse contra los huelguistas y las movilizaciones obreras.

Tras dieciséis días de paro y luego de varias reuniones mantenidas con emisarios del presidente, la dirigencia de la COB redujo el monto de los haberes originalmente solicitados a la par que acordó un cuarto intermedio de negociación. Por otra parte, como consecuencia de la protesta, Evo mandó a procesar a veintidós mineros de Huanuni acusados por la voladura del puente de Caihuasi, impulsó la revisión de las cuentas de ese yacimiento buscando suspender el control obrero colectivo y sugirió la posibilidad de que este centro laboral pudiese convertirse en una cooperativa manejada por empresarios, revirtiendo el proceso de nacionalización. ${ }^{22}$

A partir de ese último conflicto, la dirigencia de la COB dejó de sustentar su tradicional "independencia sindical" para convertirse en un aliado al gobierno. De esa manera, en forma pública, comenzó a respaldar la candidatura de Morales para las elecciones de 2014. Pese a que esta última decisión generó un sinnúmero de cuestionamientos por parte de las centrales obreras departamentales de Chuquisaca, Oruro, Beni, Potosí y de la federación de fabriles de La Paz, el apoyo al presidente se dio argumentando la "necesidad de lograr mayores espacios de poder

22 Una crónica detallada en (La Razón, La Prensa y Página Siete, mayo a septiembre de 2013). 
en la estructura del Estado" buscando la posibilidad de tener representantes en la nueva Asamblea Legislativa. ${ }^{23}$

En contrapartida a este apoyo y como forma de consolidar la alianza, Morales entregó para esa fecha un hotel con un equipamiento completo para albergar a noventa personas y dieciséis vehículos, por un valor superior al millón de dólares. ${ }^{24}$ En idéntico sentido, en señal de agradecimiento, además de llamar a respaldar la candidatura de Evo, la central obrera decidió que cada afiliado de la organización aporte con diez pesos bolivianos para la campaña electoral. En ese escenario, en las elecciones del 2014, la COB logró doce representantes en la Asamblea Legislativa Plurinacional como parte de la bancada del MAS.

A pesar de que no había pasado menos de ocho meses de haber asumido la tercera gestión, en septiembre de 2015, el MAS instaló su proyecto de presentarse para un nuevo período presidencial a partir de 2020. Cabe observar que esta iniciativa estuvo acompañada por Guido Mitma, quien por entonces ejercía el cargo de Secretario Ejecutivo de la COB, tras su designación en enero de 2016. Más aún, la central sindical como el resto de la CONALCAM escoltó a Morales en la campaña a favor del Sí en el referéndum del 21 de febrero para reformar la Constitución y permitir que se presente Evo en los comicios del 2019.

Sin embargo, la sintonía entre la dirigencia laboral y el presidente duró poco tiempo. El hecho que precipitó la ruptura fue una serie de medidas de fuerza que llevó a cabo la federación de fabriles tras el cierre de la empresa estatal ENATEX. En ese marco, luego de varios años de calma, la central laboral efectuó tres paros nacionales: de 24, 48 y 72 horas con movilizaciones y enfrentamientos con la policia. ${ }^{25}$

A la par que transcurría ese conflicto, las entidades laborales que integraban la COB comenzaron a dividirse frente a la nueva postulación electoral de Morales. Por un lado, Mitma junto con los trabajadores fabriles y diversos sindicatos adoptaron la postura de no apoyar la candidatura de Evo. Por el contrario, los mineros, los petroleros junto con la CONALCAM se abocaron a desconocer la derrota del referéndum, sumándose a la propuesta oficialista. Valga indicar que esta división en la matriz obrera continuó hasta enero de 2018 cuando en el XVII Congreso de la $\mathrm{COB}$, con una abierta intervención de Evo, se eligió a Juan Carlos Huarachi como nuevo Secretario Ejecutivo. A partir de ese momento, la dirigencia de la central se

23 Las discusiones internas se pueden seguir en (Página Siete, 30 de noviembre y 3 de diciembre de 2013).

${ }^{24}$ En la entrada del hotel se puso un busto del primer mandatario. Véase, (La Razón, 2 de mayo de 2014).

25 La Empresa Pública Nacional Estratégica de Textiles (ENATEX) fue creada por el gobierno en junio de 2012 para proteger la industria textil; sin embargo, en mayo de 2016 decidió cerrarla dejando cerca cientos de trabajadores despedidos (La Razón y Página Siete, mayo a diciembre de 2016).

\section{anuario.}


Tensiones económicas, políticas y sociales durante los gobiernos de Evo Morales. Una interpretación sobre el golpe de Estado

sumó sin vacilación a la campaña presidencial; como resultado de ello, la cúpula planteó la necesidad de aportar entre el 1\% y el 3\% de sus salarios para financiar la campaña de sus cincuenta y cuatro candidatos a diputados y senadores que se encontraban en las listas del MAS. ${ }^{26}$

Es evidente que la $\mathrm{COB}$ cambió tras el proceso de restructuración capitalista abierto en la década de 1980. A eso se añadió la emergencia de actores sociales (campesinos, indigenas) que compitieron y cuestionaron su papel de vanguardia en las luchas de esos años. Sin embargo, a pesar de esas mutaciones, la central madre siguió planteando la política en las calles a través de asambleas, bloqueos de caminos, paros, movilizaciones con explosivos, entre otras acciones. En los hechos, continuó ejerciendo una modalidad cercana al sindicalismo de presión y de negociación $\mathrm{y}$, sobre todo, mantuvo su vigencia en cuanto a guiar no solo a los trabajadores asalariados sino también a organizaciones que no pertenecen al estricto mundo laboral (juntas de vecinos, agrupamientos estudiantiles, etcétera).

En ese contexto se entiende el comportamiento de su dirigencia durante las presidencias de Morales. Los hombres que condujeron la entidad fueron conscientes del panorama (sobre todo, de sus límites) que se abrió bajo la gestión del mandatario campesino. Dentro de ese escenario, la cúpula fue adquiriendo una postura de mayor acercamiento antes que de confrontación; a pesar de que en ciertas ocasiones tuvo posiciones críticas frente a Evo, nunca llegó a romper radicalmente con el gobierno. Más aún, las únicas circunstancias que lo llevaron a enfrentarlo fue cuando las bases obreras salieron a cuestionar duramente al primer mandatario. De ese modo, analizado en una perspectiva general, la COB privilegió la instancia de negociación frente a otros caminos que pudo haber seguido.

\section{A modo de conclusión}

A pocas horas de conocerse los resultados de los comicios de octubre de 2019, el escenario que se abría en la sociedad boliviana era totalmente incierto. A diferencia de los años anteriores, no existía una firme certeza en amplios sectores de la población sobre la validez del triunfo electoral. Con el correr de las jornadas, el panorama se hizo más inestable. Por distintas razones, los antiguos aliados del mandatario campesino comenzaron no solo a alejarse, sino que también se movilizaron contra su persona. A su vez, las confusas, frágiles y contradictorias respuestas que daba Morales y sus principales allegados no calmaron las

\footnotetext{
26 En un primer momento la COB solicitó que Evo fuese acompañado en la fórmula presidencial por un obrero como vice. Sin embargo, ese pedido se diluyó y tan solo propuso sus aspirantes para la Asamblea Legislativa (Página Siete, 10 de diciembre de 2018 y 13 de agosto de 2019).
}

\section{anuario.}


demandas e incluso, en algunas circunstancias, exacerbaron los

ánimos de los opositores y dejaron sin herramientas coherentes a sus defensores. En cierto modo, en escasas semanas se había preparado el terreno para la renuncia del binomio presidencial tras el golpe de Estado encabezado por el comandante en jefe de las Fuerzas Armadas Williams Kaliman. En otras palabras, fue un derrocamiento que contó con un importante respaldo de sectores sociales y grupos corporativos que en su momento integraron y apoyaron al MAS.

Ahora bien, ¿cómo se llegó a esa situación? Sin detenernos en la reacción negativa que provocó la interrupción del escrutinio provisorio como tampoco en el desgaste que arrastraba el gobierno con su empecinado intento de lograr un nuevo mandato, existieron a la par una serie de factores coyunturales que precipitaron la renuncia. ${ }^{27}$ En parte, las respuestas se encuentran en lo que se ha observado en el presente artículo.

Por un lado, el funcionamiento que ha tenido el modelo económico sobre la base de la exportación de productos primarios ha mostrado los límites y su vulnerabilidad frente a las fluctuaciones del mercado internacional. En cierta manera, se conformó un país con una exigua diversificación del aparato productivo, que genera empleos de escasa calidad con bajos ingresos y alta precarización laboral. De manera paralela, el proyecto del MAS se enfocó en consolidar un proceso de acumulación de capital sostenido bajo pilares extractivistas, el cual, para que siga funcionando, necesita expandir las fronteras geográficas. En esa dinámica no solo entra en contradicción con el medio ambiente, sino que también lo hace con la población que habita en esos territorios. De ahí que el incremento de la expoliación de los recursos naturales -tanto por el Estado como por los capitales extranjeros y nativos- haya provocado serios resquemores y enfrentamientos contra el gobierno en los últimos años. Más aún, fueron en esos departamentos (Santa Cruz, Beni, Potosí y Chuquisaca) donde se produjeron las principales movilizaciones contra Morales en el último tiempo de su gestión. ${ }^{28}$

Por otra parte, en las semanas previas y posteriores a su renuncia, en diversos ámbitos (incluso, en el propio entorno presidencial) se esperaba una reacción más intensa a favor de su gobierno por parte de aquellos movimientos sociales que lo habian apoyado con anterioridad. Sin embargo, esto no sucedió. Como hemos observado, las organizaciones indigenas (CIDOB, CONAMAQ, entre otras) desde hacía varios años se habían alejado del MAS y de sus aliados (CONALCAM); más

\footnotetext{
$27 \mathrm{Si}$ bien no se desconoce el papel desempeñado por distintos organismos internacionales en la renuncia, en el presente apartado solo se quiere hacer énfasis en los factores domésticos.

28 Entre otras movilizaciones opositoras se puede mencionar la X marcha indigena de los pueblos orientales en defensa de la Chiquitania contra los desmontes y los incendios, los paros cívicos por el pedido de anulación del decreto que permitía la industrialización del litio en Potosí o las protestas por las regalias departamentales en las explotaciones de gas en Chuquisaca.
}

\section{anuario.}


Tensiones económicas, políticas y sociales durante los gobiernos de Evo Morales. Una interpretación sobre el golpe de Estado

aún, este proceso se había profundizado tras los hechos de corrupción con el Fondo Indígena y por el avance permanente sobre los territorios intangibles. En idéntica situación se encontró la dirigencia de la $\mathrm{COB}$, que pese a los acuerdos que mantenía con el gobierno, tuvo serias dificultades para movilizar a sus bases en defensa del mandatario cocalero. Era evidente que los afables vínculos mantenidos entre Morales y los jerarcas sindicales no reflejaban fielmente el sentir del conjunto de las entidades de base. Así lo reflejaron tanto los innumerables conflictos que emergieron por fuera de la voluntad de la dirigencia como la abierta intervención del partido oficial en la elección de Huarachi como Secretario Ejecutivo. Por otro lado, tampoco fue extraño su comportamiento si uno tiene en cuenta la larga tradición cobista de que en determinadas circunstancias recurre a su prescindencia política en función de respetar la histórica "independencia de clase".

Reflexionar sobre el final de la presidencia de Morales nos permite comprender los limites y tensiones del Proceso de Cambio. Así, se puede observar que las organizaciones sociales que en su momento lo apoyaron -sobre todo, al calor de los debates de la Asamblea Constituyente- en estos últimos tiempos dejaron de sostenerlo. En los hechos, desde mediados de su segunda presidencia Evo fue perdiendo su base social y politica, más aún, algunos se convirtieron en opositores a su mandato. Comenzó a ser evidente que la mística generada alrededor del otrora líder cocalero no era la misma. Esto en parte explica por qué no solo no se movilizaron a favor de él cuando comenzaron las manifestaciones en su contra, sino que se plegaron y marcharon (algunos con sus propias demandas) con el arco opositor. En el mismo sentido, cabe preguntarse ¿hasta dónde esos movimientos no se fueron desarticulando en su seno?; de ese modo, es válido hipotetizar como respuesta que en esto haya intervenido el fuerte crecimiento de una capa de dirigentes ligados a través de lazos clientelares al Estado, alejados de las demandas cotidianas de sus bases. En otras palabras, lo que se ha registrado es una clara pérdida de independencia de esas organizaciones a la par que se produjo una explícita cooptación por medio de prebendas.

Por último, y dejando el tema para otra ocasión, el pensamiento crítico debería analizar seriamente hasta donde el MAS ejerció una administración de corte radical y socialista. En ese plano, antes que nada, habria que sincerarse y dejar de seguir sosteniendo que éste junto con otros gobiernos que emergieron en los albores del siglo XXI tuvieron los mismos objetivos que los padres fundadores del marxismo. En esa necesaria reflexión no solo habría que detenerse en el proyecto de modernización capitalista que desarrolló, sino que también habría que pensar qué modelo de Fuerzas Armadas tuvo en mente, que ni bien tuvo la oportunidad no dudó en derrocar a su mandatario. 
Tensiones económicas, politicas y sociales durante los gobiernos de

Evo Morales. Una interpretación sobre el golpe de Estado

Alejandro M. Schneider

Anuario No 32 / ISSN 1853-8835 / 2020

http://anuariodehistoria.unr.edu.ar/ojs/index.php/Anuario/index

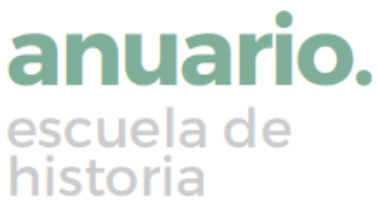

\section{Bibliografia}

Arce Catacora, L. (2011). El nuevo modelo económico, social, comunitario, productivo. Revista Economía Plural (1). Ministerio de Economía y Finanzas Públicas. La Paz: Bolivia.

Centro de Estudios para el Desarrollo Laboral y Agrario. (2009). Alerta Laboral. (59).

Centro de Estudios para el Desarrollo Laboral y Agrario. (2013). Alerta Laboral. (71).

Centro de Estudios para el Desarrollo Laboral y Agrario. (2018). Plataforma Energética. Cuadernos de Coyuntura (21).

Cortez, J. (2011). "Las connotaciones politico-institucionales del conflicto por el TIPNIS", Andamios. (3).

Díaz Cuellar, V. (2017). Ganancia, salario y renta en el sector minero en Bolivia durante el gobierno del MAS (2006-2015). En C. Arze et.al. Reporte Anual de Industrias Extractivas. La Paz: CEDLA.

Escóbar de Pabón, S., Arteaga Aguilar, W., Hurtado Aponte, G. (2019). Medición de la pobreza multidimensional Bolivia 2017. La Paz: CEDLA.

Fortún, I. (2012). TIPNIS: la verdad social oculta detrás de la coyuntura y la conflictividad. Tarija: PNUD.

Fundación UNIR (2012). La conflictividad en Bolivia. Estado de Situación. La Paz: Fundación UNIR.

Gandarillas, M. (2016). Bolivia: La década dorada del extractivismo. En: VV.AA. Rescatar la esperanza. Más allá del neoliberalismo y el progresismo. Barcelona: Entrepueblos.

García Linera, Á. (2011). El "Oenegismo", Enfermedad Infantil Del Derechismo. (O cómo la "reconducción" del Proceso de Cambio es la restauración neoliberal). La Paz: Vicepresidencia del Estado Plurinacional.

García, Linera, Á. (2012). Geopolitica de la Amazonia. La Paz: Vicepresidencia del Estado Plurinacional.

García Linera, Á. (2006). "El 'capitalismo andino amazónico", Le Monde Diplomatique, enero.

Esta obra está sujeta a la Licencia Reconocimiento-NoComercial-Compartirlgual 4.0 Internacional de Creative Commons. http://creativecommons.org/licenses/by-nc-sa/4.0/ 
Tensiones económicas, politicas y sociales durante los gobiernos de Evo Morales. Una interpretación sobre el golpe de Estado

Instituto Nacional de Estadísticas. (2014). Estadisticas económicas. La Paz.

Instituto Nacional de Estadísticas. (2018). Salario, Remuneración y Empleo del Sector Privado 2012-2017. La Paz.

Leaño, E. (2012) "Movilidad social y conflictos en Bolivia” en Andamios. (6).

Ministerio de Economía y Finanzas Públicas. (2006). Plan Nacional de Desarrollo: Bolivia digna, soberana, productiva y democrática para Vivir Bien. La Paz: Ministerio de Economía y Finanzas Públicas.

Ministerio de Economía y Finanzas Públicas. (2018). 12 Años Estabilidad Económica-Bolivia. La Paz: Editorial del Estado.

Ministerio de Minería y Metalurgia. (2013). Exportaciones mineras por empresa 2000-2012. La Paz: Ministerio de Minería y Metalurgia.

Ministerio de Planificación del Desarrollo. (2016). Plan de desarrollo económico y social en el marco del desarrollo integral para vivir bien 2016 - 2020. La Paz: Ministerio de Planificación del Desarrollo.

Ministerio de Trabajo, Empleo y Previsión Social. (2017). Plan Integral de Empleo. La Paz: Ministerio de Trabajo, Empleo y Previsión Social.

Morales Álvarez, M. (Comp.). (2015). FONDO INDÍGENA: La gran estafa. Denuncias y propuestas desde los Pueblos Indigenas. La Paz: Viejo Topo editores.

Quiroga, M. et al. (2012). Perfiles de la conflictividad social en Bolivia (2009 2011) Análisis multifactorial y perspectivas. La Paz: Fundación UNIR.

Schneider, A. (2018). Conflicto laboral, tensiones sindicales y el papel de la COB durante los dos primeros gobiernos del MAS (2006-2014). En: H. Camarero y M. Mangiantini (Comps.) El movimiento obrero y las izquierdas en América Latina. Experiencias de lucha, inserción y organización. Raleigh: Editorial A Contracorriente y North Caroline State University, Vol. 2.

Schneider, A. (2016). Economía, política y conflictividad minera durante las presidencias de Evo Morales en Bolivia (2006-2016). En Perfiles Económicos. (1).

Urioste, M. (2011). Concentración y extranjerización de la tierra en Bolivia. La Paz: Fundación Tierra. 
Wanderley, F. et.al. (2018). Hacia el desarrollo sostenible en la región andina. Bolivia, Perú, Ecuador y Colombia. La Paz: Universidad Católica Boliviana "San Pablo".

VV. AA., (2012). La MAScarada del poder: Manifiesto Junio 22. Cochabamba: Textos Rebeldes.

Yáñez, E. (2018). "Aproximación a la calidad del empleo en Bolivia (20062015)" en Rodney Pereira Maldonado (Coord.) Análisis del empleo en Bolivia. Calidad, sector gremial y actores. La Paz: Vicepresidencia del Estado Plurinacional.

Zuazo, M. (2010). ¿Los movimientos sociales en el poder? El gobierno del MAS en Bolivia. Revista Nueva Sociedad. (227).

\section{Prensa}

La Prensa, La Paz.

La Razón, La Paz.

Página Siete, La Paz. 